\title{
Exercise based assessment of cardiac autonomic function in type 1 versus type 2 diabetes mellitus
}

Jeffrey J. Goldberger ${ }^{1}$, Daniel J. Pelchovitz ${ }^{2}$, Jason $\mathrm{Ng}^{2}$, Haris Subacius ${ }^{2}$, Alexandru B. Chicos ${ }^{2}$, Smriti Banthia ${ }^{2}$, Mark Molitch ${ }^{3}$, Ronald B. Goldberg ${ }^{4}$

${ }^{1}$ Division of Cardiology, University of Miami Miller School of Medicine, Miami, FL, USA ${ }^{2}$ Division of Cardiology, Feinberg School of Medicine, Northwestern University, Chicago, IL, USA ${ }^{3}$ Division of Endocrinology, Feinberg School of Medicine, Northwestern University, Chicago, IL, USA

${ }^{4}$ Division of Endocrinology, University of Miami Miller School of Medicine, Miami, FL, USA

\begin{abstract}
Background: Cardiac autonomic neuropathy (CAN) is a complication of diabetes mellitus (DM) that is associated with increased mortality. Exercise-based assessment of autonomic function has identified diminished parasympathetic reactivation after exercise in type 2 DM. It is postulated herein, that this would be more prominent among those with type 1 DM.

Methods: Sixteen subjects with type 1 DM (age 32.9 \pm 10.1 years), 18 subjects with type 2 DM (55.4 \pm \pm 8.0 years) and 30 controls (44.0 \pm 11.6 years) underwent exercise-based assessment of autonomic function. Two 16-min submaximal bicycle tests were performed followed by 45 min of recovery. On the second test, atropine $(0.04 \mathrm{mg} / \mathrm{kg})$ was administered near end-exercise so that all of the recovery occurred under parasympathetic blockade. Plasma epinephrine and norepinephrine levels were measured at rest, during exercise, and during recovery.

Results: There were no differences in resting or end-exercise heart rates in the three groups. Parasympathetic effect on $R R$-intervals during recovery $(p<0.03)$ and heart rate recovery $(p=0.02)$ were blunted in type 2 DM. Type 1 DM had higher baseline epinephrine and norepinephrine levels $(p<0.03)$, and exhibited persistent sympathoexcitation during recovery.

Conclusions: Despite a longer duration of DM in the study patients with type 1 versus type 2 DM, diminished parasympathetic reactivation was not noted in type $1 \mathrm{DM}$. Instead, elevation in resting plasma catecholamines was noted compared to type 2 DM and controls. The variable pathophysiology for exercise-induced autonomic abnormalities in type 1 versus type 2 DM may impact prognosis. (Cardiol J 2022; 29, 2: 272-283)
\end{abstract}

Key words: cardiac autonomic neuropathy, diabetes mellitus, exercise testing, cardiac autonomic function

\section{Introduction}

Diabetes mellitus (DM) is an increasingly prevalent problem, with an estimated worldwide prevalence of 285 million people [1]. Longitudinal data from Framingham shows that approximately onefifth of sudden cardiac deaths (SCDs) occurred in the setting of DM [2]. DM independently increases cardiovascular mortality as patients with DM without prior myocardial infarction or with preserved ejection fraction have the same event rates as nondiabetic patients with prior myocardial infarction [3] or with low $(<35-40 \%)$ ejection fraction $[4,5]$, respectively. DM patients are at higher risk for SCD [6-13], even after adjustment for traditional risk factors such as coronary artery disease (CAD), hypercholesterolemia, and hypertension. The independent contribution of DM to cardiac mortality is thus well established.

Address for correspondence: Jeffrey Goldberger, MD, University of Miami Miller School of Medicine, 1120 NW $14^{\text {th }}$ Street, Suite 1124, Miami, FL 33136, USA, tel: (305) 243-4356, fax: (305) 243-7106, e-mail: j-goldberger@miami.edu

Received: 3.12.2019 Accepted: 13.04.2020 Early publication date: 7.05.2020

This article is available in open access under Creative Common Attribution-Non-Commercial-No Derivatives 4.0 International (CC BY-NC-ND 4.0) license, allowing to download articles and share them with others as long as they credit the authors and the publisher, but without permission to change them in any way or use them commercially. 
The Action to Control Cardiovascular Risk in Diabetes (ACCORD) trials demonstrated that tight control of blood glucose, lipids, and hypertension may not translate into improved survival [14-16], suggesting that other factors may be responsible for the increased mortality observed in patients with DM. Cardiac autonomic neuropathy (CAN) is a finding in DM with a prevalence that depends on the population studied and the diagnostic criteria used and is associated with an increased risk of SCD $[17,18]$, making it a potential target for treatment. The initial manifestations of CAN are thought to be due to diminished parasympathetic activity in the setting of preserved sympathetic tone $[19,20]$. Because the risk for SCD is dramatically increased during periods of sympathoexcitation, such as related to exertion [21], an exercise-based assessment of parasympathetic effect was developed [22-26]. With exercise, there is a change from vagal predominance at rest to sympathetic predominance but not an absolute withdrawal of parasympathetic activity [24, 27]. A technique for exercise-based assessment uses parasympathetic blockade with atropine to assess the RR-interval in the presence and absence of cardiac parasympathetic input. With the exercise-based methodology, abnormalities in parasympathetic reactivation after exercise were identified in subjects with type $2 \mathrm{DM}$ without overt CAN, as defined by the current diagnostic criteria [20]. Importantly, this abnormality appeared to be specific to diabetes, as it was not observed in a prior study of patients with CAD, even those with left ventricular dysfunction [26]. It was also demonstrated that the directional change in the QT interval (otherwise known as QT-RR hysteresis) is driven by changes in the parasympathetic nervous system during exercise and recovery [28], highlighting the role of the parasympathetic inputs on cardiac repolarization and providing a potential pathophysiologic link among diabetics, CAN, and the increased risk of SCD with exercise.

It was therefore hypothesized herein, that an exercise-based assessment of cardiovascular autonomic inputs would also identify these abnormalities in asymptomatic subjects with type 1 diabetes, a population that has a higher rate of moderate to severe CAN than subjects with type 2 diabetes [29] and with longer resting QTc [30]. In addition, it was hypothesized that the extent and severity of CAN would differ between subjects with type 1 and type 2 diabetes given the differences in extent/duration of hyperglycemia in these patients, specifically that the population with type 1 diabetes would exhibit a more severe phenotype than those with type 2 diabetes, and that the primary abnormality would be a further reduction in parasympathetic effects during exercise and recovery than was demonstrated previously in subjects with type 2 diabetes using an exercise-based assessment of autonomic function.

\section{Methods}

\section{Subjects}

Group I included 30 healthy volunteers aged $18-70$ years without a diagnosis of DM. All subjects in this group were free from significant medical conditions, were not taking cardioactive medications, and had normal physical examinations and electrocardiograms (ECG). Several of these volunteers were controls in a previous publication [20]. Group II included 16 volunteers with type $1 \mathrm{DM}$ as defined by the American Diabetes Association report criteria and were phenotyped as having type $1 \mathrm{DM}$ by experienced diabetologists [31]. Subjects were included if they had diabetes for $>1$ year, were on a stable insulin regimen for $>3$ months, participated in aerobic exercise for $>60 \mathrm{~min} /$ /week, and weighed $<90 \mathrm{~kg}$ and were excluded if they were taking beta-blockers. Subjects with autonomic disorders, myocardial ischemia/infarction, heart failure, arrhythmias, and pacemakers were excluded. Patients with neuropathy, nephropathy, microalbuminuria, retinopathy, or systemic illnesses such as asthma and renal insufficiency were also excluded. For comparison purposes, Group III included 18 subjects with type $2 \mathrm{DM}$ who were previously studied and reported on [20]. Subjects in this group had DM > 1 year, were on a stable medical regimen for 3 months. None were taking insulin. As in group II, these subjects were required to participate in regular cardiovascular exercise and could not be on beta-blocker medications. The study protocol for these subjects was identical to the current report, allowing a comparison of results in subjects with type 1 and type 2 diabetes.

\section{Peripheral neuropathy and CAN testing}

All subjects with a diagnosis of DM were screened with the Michigan Neuropathy Screening Instrument (MNSI) to assess for peripheral neuropathy on study visit one [32]. CAN was assessed this same day with a battery of 5 noninvasive cardiovascular reflex tests [33]. The tests, as previously described, included heart rate response to deep breathing, standing, and Valsalva maneuver, as well as blood pressure response to standing and sustained handgrip. Results of these tests are 
referred to as the Ewing score; higher scores indicate more severe CAN. While subject enrollment was restricted to those without clinical evidence of peripheral neuropathy or CAN, the MNSI or Ewing score was not used to exclude subjects.

\section{Exercise testing}

All subjects underwent seated bicycle exercise testing on 2 days separated by $\geq 72 \mathrm{~h}$. Subjects continued their usual insulin regimen (for those on insulin) and diet prior to the test and were asked to refrain from caffeine prior to the test. Subjects with insulin infusion pumps continued their usual insulin protocol in order to avoid hypoglycemia. When possible, subjects were studied at the same time of day. An intravenous catheter was inserted into an antecubital vein for blood draws and/or drug administration. Subjects were attached to an ECG machine (Burdick Quest Exercise Stress System, Cardiac Science, Bothell, WA). For subjects with diabetes, a blood sample was drawn for measurement of hemoglobin A1c, blood urea nitrogen, and creatinine levels. For all subjects, blood was drawn for measurement of plasma catecholamines at rest after assuming a seated resting position for $\geq 5 \mathrm{~min}$. All subjects had normal resting blood pressures. All measurements were made with subjects seated on an electrically-braked bicycle ergometer (SciFit ProII, Tulsa, OK). Continuous 12-lead ECG monitoring was performed for a 5 -min rest period, a 16-min exercise period, and a 45-min recovery period. Subjects were instructed to maintain a pedal speed of $80 \mathrm{rpm}$ during all phases of the exercise session. The exercise protocol began at a 50-Watt workload, increased to 75 Watts at $4 \mathrm{~min}$ and 100 Watts at 6 min, as tolerated. Subjects continued to exercise at this workload for an additional $10 \mathrm{~min}$. At the end of 16 min of exercise, heart rate and blood pressure were recorded, and exercise was stopped. All subjects demonstrated the expected increase in heart rate and blood pressure with exercise with no evidence of ischemia. Blood samples were drawn for plasma catecholamine levels at 8 and 15 min of exercise, and minutes $5,10,20,30$, and 45 of recovery.

On the second session, the identical protocol was performed. However, intravenous atropine $(0.04 \mathrm{mg} / \mathrm{kg})$ was administered in 4 divided doses $(0.01 \mathrm{mg} / \mathrm{kg}$ every $30 \mathrm{~s})$ starting at $12 \mathrm{~min}$ of exercise to achieve complete parasympathetic blockade. Parasympathetic blockade with atropine has a rapid onset, has been shown previously to last over $1 \mathrm{~h}$, and is considered the gold standard for evaluation of parasympathetic effects [34, 35]. To ensure subject safety and ability to complete the exercise protocol, the first session was always performed without parasympathetic blockade and the second session was performed with parasympathetic blockade.

\section{Parasympathetic effect}

For each subject and at each time point in recovery, parasympathetic effect on the RR-interval was defined as the difference of the RR-interval $(\Delta \mathrm{RR})$ at baseline without atropine and the RR-interval after parasympathetic blockade with atropine.

\section{Plasma catecholamines}

Blood samples were collected for epinephrine and norepinephrine (NE) levels in heparinized tubes and placed on ice. After centrifugation at $4^{\circ} \mathrm{C}$ at $3000 \mathrm{rpm}$ for $15 \mathrm{~min}, 2 \mathrm{~mL}$ of plasma was transferred to an empty tube and stored at $-70^{\circ} \mathrm{C}$ for subsequent analysis. This sample was then transferred to Quest Diagnostics Laboratories with dry ice for analysis, as previously reported [20].

\section{RR-intervals and heart rate recovery}

Electrocardiogram data were analyzed with custom software (MATLAB-Mathworks, Natick, MA). Using a QRS template-matching algorithm, an interval tachogram was generated from the continuous ECG recording. All recordings were examined and manually overread to verify beat classification. Early heart rate recovery (EHRR) was calculated as end-exercise, exercise heart rate minus heart rate at 1 min of recovery. Late heart rate recovery was calculated as RR-interval at 5 min of recovery minus end-exercise RR-interval divided by RR-interval at rest minus RR-interval at end-exercise multiplied by 100 [36].

\section{Heart rate variability}

Heart rate variability analysis was performed on 5 min resting ECG recordings obtained prior to the initiation of exercise. The time domain parameters of root mean square of successive differences and root mean square residuals of linearly detrended RR-intervals (RMS) were calculated for five consecutive 1-min periods during the initial resting period [37]. The five values were averaged for analysis.

Frequency domain analysis was performed. The RR-intervals from these recordings were resampled at $4 \mathrm{~Hz}$ and then linearly detrended. After applying a Hanning window, the fast Fourier transform was used to obtain the power spectrum. Low frequency (LF) power was measured in the 0.04 to $0.15 \mathrm{~Hz}$ band while high frequency (HF) power was measured in the 0.15 to $0.5 \mathrm{~Hz}$ band [38]. 


\section{QT interval and indices}

\section{of cardiac repolarization}

QRS onset was identified using slope threshold criteria. T wave offset was estimated as the point where the tangent of the maximum descending $\mathrm{T}$ wave slope intersected the isoelectric line. The median values of all leads were chosen as the global QRS onsets and T wave offsets for each beat from which QT intervals were calculated. All measurements were visually confirmed.

Because of the changing heart rate throughout the protocol and differences between subject heart rates, comparison of cardiac repolarization parameters is challenging and a gold standard is lacking. We therefore calculated the QT-RR slope in recovery, which has been shown to be a more reliable description of repolarization in the setting of changing heart rates as compared to the QT interval [39]. The QT-RR slope was calculated for each subject using linear regression analysis from 6 QT-RR-interval pairs $(0-5 \mathrm{~min})$ in early recovery, as this has been shown to have prognostic significance [40]. Baseline QTc values were also calculated using the Bazett formula.

\section{Statistics}

Comparisons between groups were performed with analysis of variance for continuous variables and $\chi^{2}$ tests for categorical variables. Data are presented as mean \pm standard error and counts (\%) in the demographic tables and for non-modeled data, including heart rate variability, baseline heart rates, end-exercise heart rates, heart rate recovery, and baseline QTc. Natural logarithm transformation was used for NE data to better approximate normality.

A repeated-measures mixed-effects model with random effects of subjects was used to analyze all other outcome data including parasympathetic effect, epinephrine level, NE level, and QT-RR slope. First-order auto-regressive variance-covariance structure was used to model the correlations among measurements in time. Because of the age and sex differences among the groups, these factors were included in the analyses of autonomic effects. Thus, the fixed effects included age, sex, type of diabetes, parasympathetic blockade, their interaction, as well as time in recovery. As appropriate, exercise, early recovery and late recovery segments of the data were analyzed in separate independent models. In the presence of interactions with sex, males and females were also analyzed in separate models. A single extreme positive outlier value of epinephrine $(>600 \mathrm{pg} / \mathrm{mL})$ was excluded from analyses. ANOVA was used to compare baseline characteristics among the three study groups where there are no measurements clustered within patients. Mixed effects models, on the other hand, were used in all cases with repeated measurements across time within patients. A p-value $<0.05$ was considered significant. Modeled effects are reported as mean \pm standard error. All modeled effects are reported with adjustment for sex and age. Results for $\mathrm{NE}$ are represented as 95\% confidence intervals (CI) as the model used logarithmic transformation.

\section{Study approval}

The study was approved by the Northwestern University Institutional Review Board. Written informed consent was required from all subjects prior to enrollment in the study.

\section{Results}

\section{Baseline characteristics}

Baseline characteristics are shown in Table 1. The 16 subjects with type $1 \mathrm{DM}$ (4 male, age 32.9 \pm 10.2 years) were significantly younger than the 18 subjects with type $2 \mathrm{DM}$ that were previously studied [20] (12 male, age $55.4 \pm 8.0$ years). There were 30 controls ( 15 male, age $44.0 \pm 11.6$ years). Subjects with type 1 diabetes had a mean hemoglobin A1c of $7.1 \pm 0.9 \%$ and average duration of diabetes of $18.6 \pm 7.6$ years, while subjects with type 2 diabetes had a mean hemoglobin A1c of $6.4 \pm$ $\pm 0.7 \%(\mathrm{p}<0.02)$ and average duration of diabetes of $5.3 \pm 4.0$ years $(\mathrm{p}<0.01)$. All subjects with type 1 diabetes were on an insulin regimen, while no subject with type 2 diabetes was on insulin and 16 of the 18 subjects (89\%) were taking metformin. Subjects with diabetes had no clinical evidence of peripheral or autonomic neuropathy and had low overall scores on CAN testing (Ewing score $0.8 \pm 0.8$ for type $1 \mathrm{DM}$ and $1.2 \pm 0.9$ for type 2 $\mathrm{DM}, \mathrm{p}=\mathrm{NS})$. Only 1 subject with type 1 diabetes demonstrated significant resting hypoglycemia on one of the visits. One subject with type $2 \mathrm{DM}$ and 4 subjects with type $1 \mathrm{DM}$ did not return for a second study visit and therefore data requiring two visits were excluded from analysis.

\section{Resting heart rate variability}

Root mean square of successive differences in type $2 \mathrm{DM}(18.8 \pm 9.4 \mathrm{~ms})$ was lower than controls $(29.4 \pm 15.1 \mathrm{~ms}, \mathrm{p}=0.04)$ and type $1 \mathrm{DM}(34.2 \pm$ $\pm 20.7 \mathrm{~ms}, \mathrm{p}=0.09)$. Similarly, RMS was lower in type $2 \mathrm{DM}(24.3 \pm 10.0 \mathrm{~ms}, \mathrm{p}=0.03)$ than controls 
Table 1. Subject characteristics.

\begin{tabular}{|c|c|c|c|}
\hline & $\operatorname{DM} 1(n=16)$ & DM 2 (n = 18) & Control $(n=30)$ \\
\hline Age [years] & $32.9 \pm 10.2$ & $55.4 \pm 8.0$ & $44.0 \pm 11.6$ \\
\hline Male & $25 \%$ & $67 \%$ & $50 \%$ \\
\hline Hypertension & $25 \%$ & $22 \%$ & $0 \%$ \\
\hline Hyperlipidemia & $25 \%$ & $78 \%$ & $10 \%$ \\
\hline Caucasian & $94 \%$ & $83 \%$ & $60 \%$ \\
\hline Duration of diabetes [years] & $18.6 \pm 7.6$ & $5.3 \pm 4.0$ & NA \\
\hline MNSI Physical & $0.08 \pm 0.28$ & $0.17 \pm 0.18$ & NA \\
\hline MNSI History & $0.46 \pm 0.52$ & $0.44 \pm 0.41$ & NA \\
\hline Ewing score & $0.78 \pm 0.82$ & $1.19 \pm 0.94$ & NA \\
\hline Weight $[\mathrm{kg}]$ & $73.7 \pm 8.0$ & $84.2 \pm 8.3$ & $76.2 \pm 14.1$ \\
\hline Body mass index $\left[\mathrm{kg} / \mathrm{m}^{2}\right]$ & $25.8 \pm 3.2$ & $28.5 \pm 3.3$ & $25.8 \pm 3.0$ \\
\hline Creatinine [mg/dL] & $0.72 \pm 0.09$ & $0.99 \pm 0.25$ & NA \\
\hline Hemoglobin A1c [\%] & $7.1 \pm 0.9$ & $6.4 \pm 0.7$ & NA \\
\hline \multicolumn{4}{|l|}{ Medications: } \\
\hline Insulin & $100 \%$ & $0 \%$ & $0 \%$ \\
\hline Metformin & $0 \%$ & $89 \%$ & $0 \%$ \\
\hline ACEI & $19 \%$ & $22 \%$ & $0 \%$ \\
\hline ARB & $13 \%$ & $6 \%$ & $0 \%$ \\
\hline ASA & $6 \%$ & $56 \%$ & $0 \%$ \\
\hline Statin & $31 \%$ & $67 \%$ & $7 \%$ \\
\hline
\end{tabular}

ACEI — angiotensin converting enzyme inhibitor; ARB - angiotensin receptor blocker; ASA — acetylsalicylic acid; DM 1 - type 1 diabetes mellitus; DM 2 - type 2 diabetes mellitus; MNSI — Michigan Neuropathy Screening Instrument

$(34.6 \pm 13.1 \mathrm{~ms})$ and type $1 \mathrm{DM}(39.6 \pm 19.8 \mathrm{~ms}$, $\mathrm{p}=0.01$ ). However, when adjusted for age and sex these differences were not significant.

Frequency domain analysis revealed that LF power was higher in type $1 \mathrm{DM}\left(433 \pm 99 \mathrm{~ms}^{2}\right.$; $\mathrm{p}=0.0015$ by ANOVA) than in type $2 \mathrm{DM}(110 \pm$ $\left.\pm 26 \mathrm{~ms}^{2} ; \mathrm{p}=0.0004\right)$ and controls $\left(223 \pm 38 \mathrm{~ms}^{2}\right.$; $\mathrm{p}=0.009)$. There was no statistically significant difference in HF power among groups $\left(36 \pm 8 \mathrm{~ms}^{2}\right.$ in type $1 \mathrm{DM} ; 16 \pm 5 \mathrm{~ms}^{2}$ in type $2 \mathrm{DM} ; 44 \pm 18 \mathrm{~ms}^{2}$ in controls).

\section{Heart rate response to exercise}

Baseline heart rates were $73.1 \pm 11.4 \mathrm{bpm}$ in type 1 diabetes, $75.0 \pm 11.2 \mathrm{bpm}$ in type 2 diabetes, and $70.1 \pm 6.0 \mathrm{bpm}$ in controls. Subjects with type 1 diabetes exercised to a workload of $90.6 \pm$ \pm 18.0 Watts, achieving a heart rate of $128.3 \pm 16.9$ $\mathrm{bpm}$. Subjects with type 2 diabetes exercised to a workload of $91.7 \pm 12.1$ Watts, achieving a heart rate of $128.9 \pm 22.0 \mathrm{bpm}$. Controls exercised to a workload of $94.0 \pm 15.9$ Watts, achieving a heart rate of $127.0 \pm 19.2 \mathrm{bpm}$. After parasympathetic blockade with atropine, end-exercise heart rate increased to $148.0 \pm 13.2 \mathrm{bpm}$ in type 1 diabetes,
$145.6 \pm 21.1 \mathrm{bpm}$ in type 2 diabetes and $149.4 \pm$ $\pm 14.8 \mathrm{bpm}$ in controls. None of these parameters differed among groups.

\section{Parasympathetic effect on the RR-interval}

RR-intervals for diabetes (type 1 and type 2) and control groups during exercise and recovery, with and without parasympathetic blockade, are shown in Figure 1A. As previously reported, subjects with type 2 diabetes had a significantly shorter average RR-interval during recovery versus controls. After parasympathetic blockade with atropine was achieved, the recovery RR-intervals curves are superimposable for the three groups.

The parasympathetic effect, $\Delta R R$, in recovery is shown in Figure 1B and Table 2. When adjusted for age and sex, the increase in parasympathetic effect $(\Delta R R)$ in early recovery was blunted in type 2 diabetes compared to type 1 diabetes ( $\mathrm{p}=$ $=0.03)$ and controls $(\mathrm{p}<0.004)$, with no difference between type 1 diabetes and controls $(p=$ $=0.78)$. In later recovery $(\geq 5 \mathrm{~min})$, when adjusted for age and sex, the type 2 diabetes group had lower parasympathetic effect than controls $(\mathrm{p}<0.002)$ with a trend for lower $\Delta \mathrm{RR}$ than type 1 diabetes 


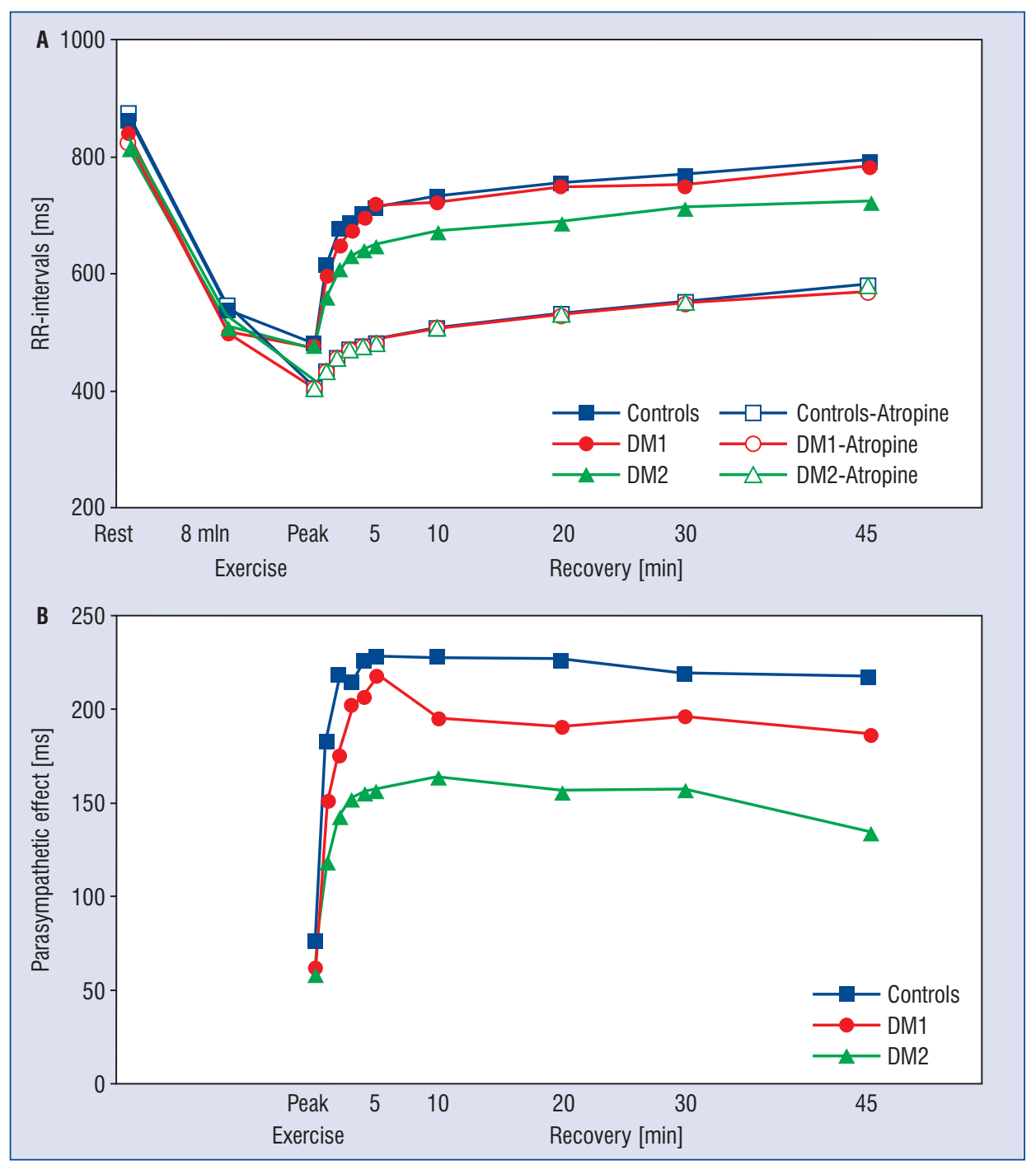

Figure 1. A. RR-intervals are demonstrated at rest, during exercise, and in recovery for each group during the initial baseline test and the second test with administration of atropine. With exercise, RR-intervals decrease in each group, and with recovery RR-intervals increase in each group. RR-intervals are shortest in the type 2 diabetes group during recovery. With atropine, the differences between groups are abolished, and RR-interval increase during recovery is blunted; the curves overlap for the three groups; B. Parasympathetic effect on the RR-intervals is shown at end exercise and in recovery for each group, defined as the difference of the RR-interval $(\triangle R R)$ at baseline without atropine and the RR-interval after parasympathetic blockade with atropine. Parasympathetic effect on RR-intervals during recovery is blunted in type 2 diabetes compared to controls and type 1 diabetes; DM - diabetes mellitus.

( $\mathrm{p}=0.07)$ and no difference between type 1 diabetes and controls $(\mathrm{p}=0.53)$. Modeled estimates for $\Delta R R$ were $224.3 \pm 12.7 \mathrm{~ms}$ for controls, $208.2 \pm$ $\pm 23.0 \mathrm{~ms}$ for type 1 diabetes, and $145.6 \pm 20.2 \mathrm{~ms}$ for type 2 diabetes.

\section{Plasma catecholamines}

Figure 2 shows the change in NE levels over time. Baseline NE levels were the highest for the type 1 diabetes group $(800.6 \mathrm{pg} / \mathrm{mL}, 95 \%$
CI 646.8-990.8 pg/mL) compared to both type 2 diabetes group (567.3 pg/mL, 95\% CI 464.0-693.5 $\mathrm{pg} / \mathrm{mL}, \mathrm{p}<0.03)$ and controls $(524.3 \mathrm{pg} / \mathrm{mL}, 95 \%$ CI $449.1-612.0 \mathrm{pg} / \mathrm{mL}, \mathrm{p}<0.01)$. There was no difference between controls and subjects with type 2 diabetes. Age had no effect on either baseline NE levels or NE change during exercise $(p>0.20)$; however, there was an interaction with sex $(p=0.02)$. There were no significant differences in end-exercise NE levels or NE increase 


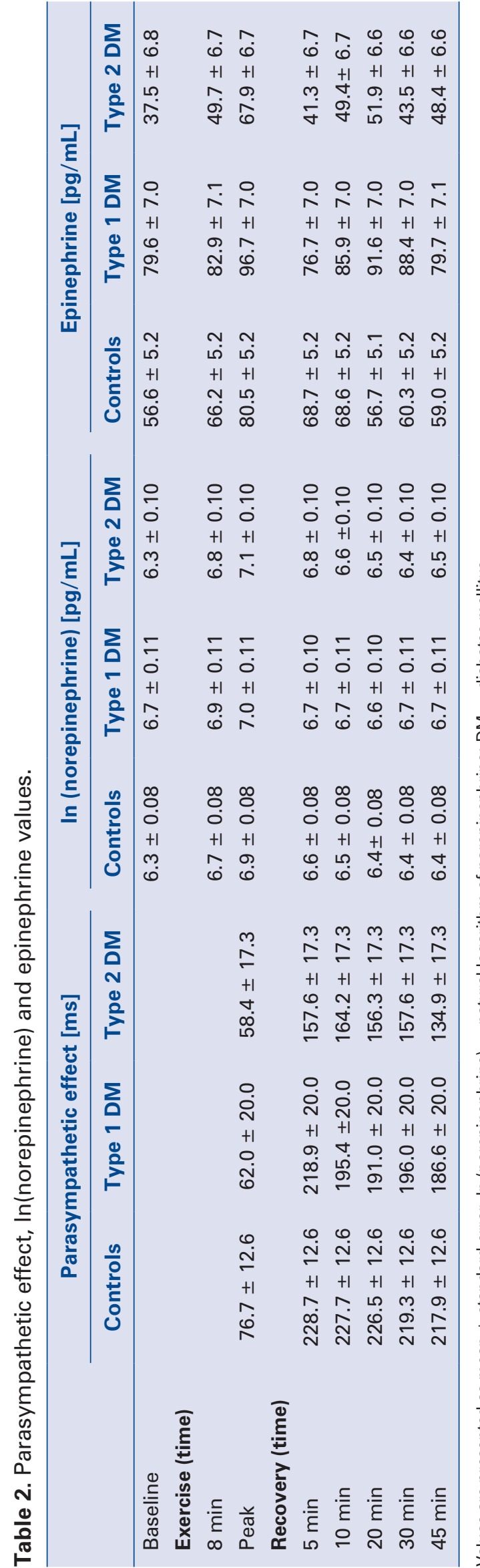

or recovery rates among the groups. When adjusted for age and sex, NE levels in later recovery (after 10 min recovery) remained elevated in type $1 \mathrm{DM}$ compared to controls $(\mathrm{p}<0.01)$ and type $2 \mathrm{DM}(\mathrm{p}<0.03)$. There was no significant difference between subjects with type 2 diabetes and controls.

Figure 2 also shows the change in epinephrine levels over time. Baseline epinephrine levels were elevated in type 1 diabetes $(79.6 \pm 7.0 \mathrm{pg} /$ $/ \mathrm{mL})$ compared to controls $(56.6 \pm 5.2 \mathrm{pg} / \mathrm{mL}$, $\mathrm{p}<0.03)$ and type $2 \mathrm{DM}(37.5 \pm 6.8 \mathrm{pg} / \mathrm{mL}$, $\mathrm{p}<0.01)$. There were no significant differences in end-exercise epinephrine levels or epinephrine increase or recovery rates among the groups. When adjusted for age and sex, epinephrine levels in later recovery (after 10 min recovery) remained elevated in type $1 \mathrm{DM}$ compared to controls $(\mathrm{p}<0.01)$ and type 2 DM $(\mathrm{p}<0.01)$. There was no significant difference between type $2 \mathrm{DM}$ and controls.

\section{Heart rate recovery}

Early heart rate recovery was highest in controls $(26.4 \pm 8.1 \mathrm{bpm})$, then in type 1 diabetes $(24.3 \pm 10.0 \mathrm{bpm})$ followed by type 2 diabetes $(19.1 \pm 8.2 \mathrm{bpm})$. After adjustment for age and sex, EHRR was significantly lower only in type 2 diabetes compared to controls $(\mathrm{p}=0.02)$. EHRR $<12$ bpm was noted in 1 control, 3 subjects with type 1 diabetes, and 2 subjects with type 2 diabetes. Late heart rate recovery did not significantly differ among type 1 diabetes $(64.5 \pm 16.9 \%)$, controls $(62.5 \pm 16.9 \%)$, or type 2 diabetes $(53.1 \pm 18.6 \%$, $\mathrm{p}=0.14$ for controls vs. type 2 diabetes).

\section{QT interval and QT-RR relationship}

QT intervals over time are shown in Figure 3. Baseline QTc did not differ among the groups: $412 \pm 19 \mathrm{~ms}, 404 \pm 19 \mathrm{~ms}$, and $402 \pm 21 \mathrm{~ms}$ in type 1 diabetes, type 2 diabetes, and controls, respectively. The QT-RR slopes during early postexercise recovery, calculated from 6 QT-RR pairs from $0-5$ min of recovery were similar between all three groups (type $1 \mathrm{DM} 0.26 \pm 0.02$, type $2 \mathrm{DM}$ $0.25 \pm 0.02$, control $0.23 \pm 0.02$ ).

\section{Discussion}

In this study, despite the substantially longer duration of diabetes in the study patients with type 1 (18.6 years) versus type 2 (5.3 years) diabetes, not only was the previously observed reduction in parasympathetic reactivation that was noted in 


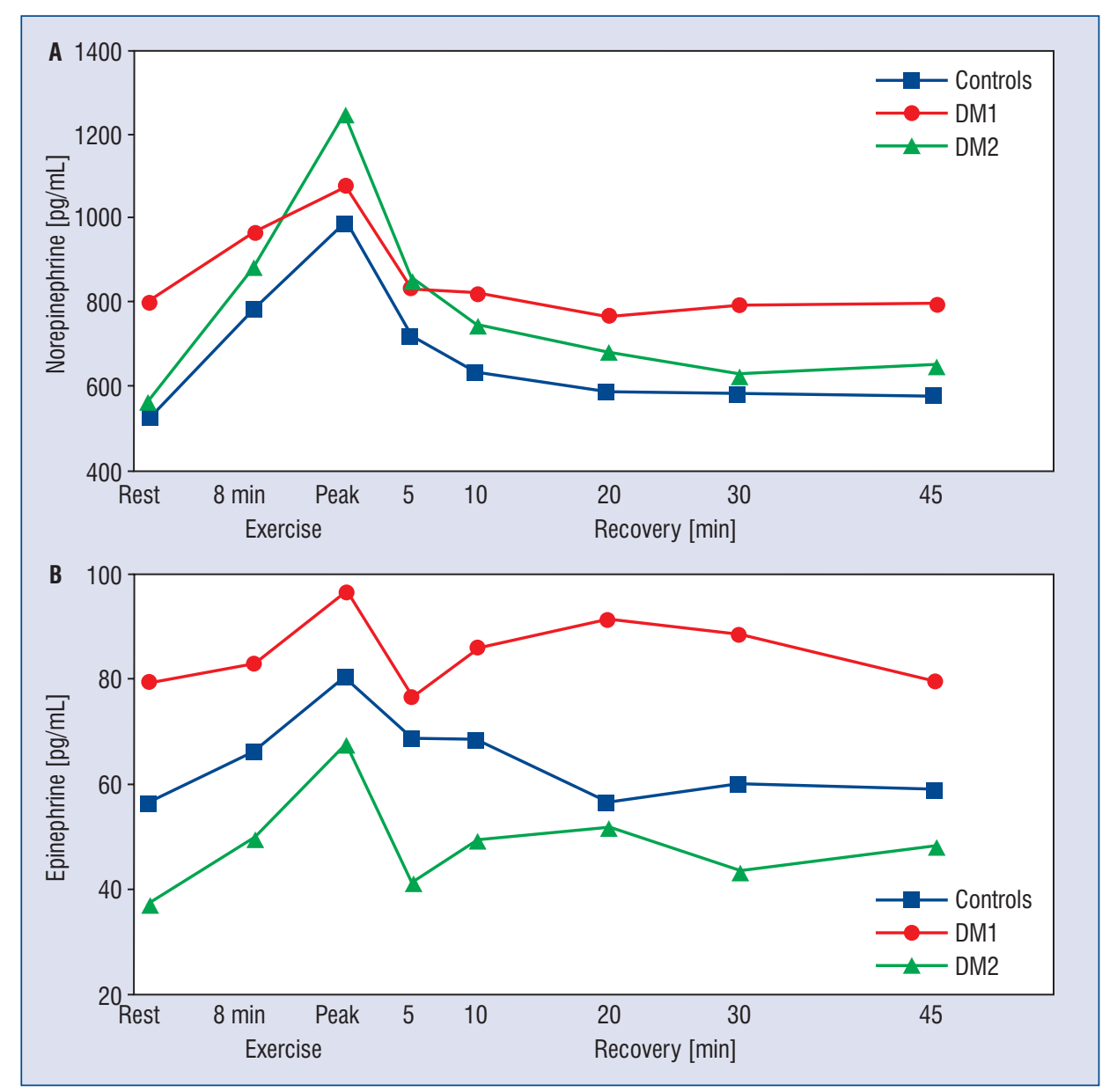

Figure 2. Norepinephrine (A) and epinephrine (B) during rest, exercise, and recovery. Plasma norepinephrine and epinephrine concentrations at rest, during exercise, and in recovery for each group. Resting norepinephrine concentration is highest in those with type 1 diabetes. With exercise, norepinephrine increases in each group, however the decrease in norepinephrine is blunted in the type 1 diabetes group compared to the type 2 diabetes group and controls. Resting epinephrine concentration is highest in type 1 diabetes. With exercise, epinephrine increases in each group, however the decrease in epinephrine is blunted in the type 1 diabetes group compared to the type 2 diabetes group and controls; DM — diabetes mellitus.

type 2 diabetes not more prominent (as hypothesized), it was in fact absent. Instead, a significant basal increase in plasma catecholamines was noted compared to type 2 diabetes and controls. The fundamental differences in the autonomic profiles uncovered by this exercise-based assessment in these populations of patients with diabetes and minimal to no evidence of clinical, peripheral, or cardiac autonomic neuropathy are clear. If these exercise-based assessments apply more broadly to $\mathrm{CAN}$ in diabetes, this raises the possibility that the pathophysiology of CAN may not be uniform in subjects with type 1 versus type 2 diabetes. While these abnormalities may produce a similar global shift in autonomic balance, the diagnostic, therapeutic, and prognostic implications of these heretofore undescribed differences require further exploration.

\section{Autonomic function}

In this study, several measures of autonomic function were assessed to delineate the spectrum of autonomic function changes observed in the population with diabetes. Resting measures included heart rate variability which evaluates the autonomic nervous system in the baseline state, a state of parasympathetic predominance. Reflex measures included the standard Ewing maneuvers which are designed to perturb the autonomic nervous system around an equilibrium point. Exercise measures, including plasma catecholamines, evaluate the autonomic nervous system during exercise, 


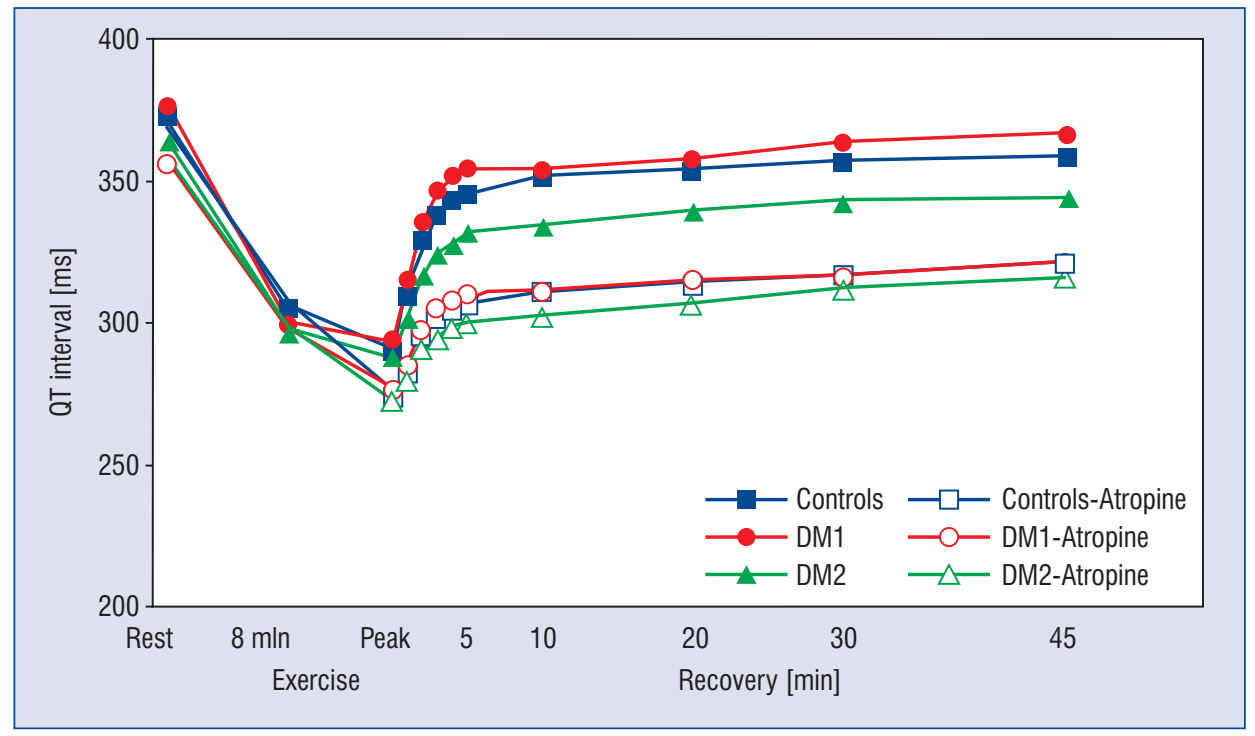

Figure 3. QT intervals over time. QT intervals are demonstrated at rest, during exercise, and in recovery for each group during the initial baseline test and the second test with administration of atropine toward the end of exercise. QT intervals decreased with exercise, and increased during recovery. QT intervals were shortest in the type 2 diabetes group during recovery. With atropine, the difference among groups was attenuated.

a time of sympathetic predominance and reduced parasympathetic activation. During recovery, parasympathetic effect and plasma catecholamines were assessed at a time of declining sympathetic effect and parasympathetic reactivation. This represents a broad snapshot of autonomic function across a spectrum of conditions. The currently, most clinically applied methodology for evaluation of CAN includes only the resting and reflex tests. The exercise testing approach used in this study represents a broader dynamic range of autonomic function and appears to be more sensitive for the detection of autonomic abnormalities in diabetes. Importantly, this exercise-based protocol was able to identify specific autonomic abnormalities that differed by diabetes type.

\section{Early CAN and ventricular repolarization in diabetes}

This study demonstrated that subjects with type 1 diabetes exhibit increased sympathetic activation as manifest by elevated catecholamine levels while those with type 2 diabetes exhibit deficient parasympathetic reactivation after exercise as manifest by decreased parasympathetic effect on $\mathrm{RR}$-intervals during recovery from exercise and decreased heart rate recovery. As the autonomic nervous system is characterized by the interplay of sympathetic and parasympathetic inputs, it is possible that the end result of either of these abnormalities is lengthening of ventricular repolarization (i.e. the QT interval), yet this was not observed in this study.

While autonomic abnormalities were detectable in both type 1 and type 2 diabetes in the present study, these were not clinically apparent as this study, by design, enrolled a relatively healthy population of patients with diabetes. Specifically, all groups had similar and fairly normal resting heart rate and chronotropic response to exercise. The only abnormality detectable by routine exercise testing was diminished early heart rate recovery in subjects with type 2 diabetes. This suggests that the body's compensatory mechanisms are able to accommodate for these early abnormalities, keeping them clinically silent. It is interesting that despite clear differences in sympathetic and parasympathetic function in the two groups of diabetics in this study, there were no clear differences in the resting QTc or recovery QT-RR relationships. This supports the notion that at this early stage, counterregulatory changes are occurring to maintain a relatively normal relationship. If this hypothesis can be verified, early treatment to prevent the progression of CAN may prevent or delay the development of cardiac repolarization abnormalities. Thus, early identification of these abnormalities could have prognostic and therapeutic significance. More importantly, it could provide a target for early treatment to prevent progression of disease and 
improve outcomes. A number of interventions have been associated with prevention and even reversal of early CAN [41-43], however this reversibility is not present in advanced disease.

\section{Pathophysiology of CAN in diabetes}

The pathophysiology of CAN in type 1 and type $2 \mathrm{DM}$ has not been differentiated. Autonomic neuropathy has been thought to be due to hyperglycemic damage to the nerves, and CAN in type 1 and type $2 \mathrm{DM}$ has been thought to be due to the same pathophysiology, specifically that longstanding hyperglycemia leads to direct toxicity to the autonomic nerves. This concept led to the hypothesis that the study patients with type 1 DM would have more prominent abnormalities in parasympathetic reactivation after exercise due to the longer duration of diabetes compared to a previously studied population with type $2 \mathrm{DM}$. This study clearly refutes this hypothesis. While it is possible to speculate that patients with type 2 DM may have more subclinical cardiac disease which is responsible for the decline in parasympathetic effect, it should be noted that in prior studies no significant decline in parasympathetic effect was noted in patients with known CAD, with or without left ventricular dysfunction [26]. Thus, the reduced parasympathetic effect in type $2 \mathrm{DM}$ is likely a specific finding for type $2 \mathrm{DM}$. The noted abnormalities in plasma catecholamines in the absence of a reduction in parasympathetic reactivation in type $1 \mathrm{DM}$ raises the question about whether these entities have differing pathophysiology for the early phases of CAN. Further study is needed to more clearly differentiate CAN in subjects with type 1 and type 2 diabetes.

\section{Potential clinical implications}

Abnormalities in cardiac autonomic activity have been implicated in myriads of studies on risk for death and SCD. Moreover, diabetes is also independently associated with SCD risk [44]. Longitudinal data from Framingham show that approximately one-fifth of SCDs occurred in the setting of DM [2]. Patients with DM are at higher risk for SCD [6-13], even after adjustment for traditional risk factors such as CAD, hypercholesterolemia, and hypertension. Given the known cardioprotective effects of parasympathetic tone and the adverse effects of sympathoexcitation, either of the abnormalities identified in the current study could theoretically contribute to the enhanced risk associated with diabetes. It is interesting that Treatment Of Preserved Cardiac function heart failure with an Aldosterone antagonist (TOPCAT) [45] identified diabetes as a risk factor for SCD in patients with heart failure and preserved ejection fraction, but this excess risk was only noted in insulin treated diabetics. Insulin has been shown to lead to increased sympathetic activity in both lean and obese subjects [46] and in animal studies [47]. In healthy women, insulin infusion during a euglycemic clamp resulted in an increase in the LF/HF ratio consistent with sympathoexcitation. These data support the notion that either insulin use or type 1 diabetes may promote adverse sympathoexcitation that specifically increases the risk for SCD.

\section{Limitations of the study}

Limitations of the current study include the small sample size of the groups, as well as the inclusion/exclusion criteria that selected for a healthier group of subjects with diabetes. Age and sex differences among the groups were noted, but are unlikely to explain the marked differences. It is notable that the groups performed exercise to similar workloads and peak heart rate. Most importantly, the absence of abnormal parasympathetic reactivation in the study patients with type 1 diabetes remains a clear, unexpected, and novel observation. In addition, parasympathetic effect is calculated as the difference in RR-intervals between the baseline state and with atropine administration. These tests occurred on separate days and therefore there may be subtle differences that cannot completely be attributed to parasympathetic influence. The basic assumption for these studies is that the exercise response to a given workload is prototypical for any individual subject and that deviations in heart rate induced by selective autonomic blockade provides information on the autonomic contribution at that time. This is confirmed by the multiple studies [22-26] performed by the present group of researchers showing reproducible heart rate trends to various stages of exercise on different days prior to the administration of autonomic blockade.

\section{Conclusions}

In conclusion, the present subjects with type 1 and type 2 diabetes exhibited different abnormalities in exercise-based autonomic profiles. In this study, type 1 diabetes was characterized by increased sympathoexcitation in the basal state and during recovery from exercise, while type 2 diabetes was characterized by decreased parasympathetic reactivation after exercise. This study suggests that CAN may differ between type 1 and type 2 
diabetes. Further study is needed to determine the origin of these differences and whether this has prognostic or therapeutic implications, particularly since cardiac autonomic neuropathy is associated with increased mortality in patients with diabetes.

\section{Funding}

This research was supported, in part, by the National Heart, Lung, and Blood Institute (Grant No. 1 RO1 HL 70179-01A2).

\section{Conflict of interest: None declared}

\section{References}

1. Shaw JE, Sicree RA, Zimmet PZ. Global estimates of the prevalence of diabetes for 2010 and 2030. Diabetes Res Clin Pract. 2010; 87(1): 4-14, doi: 10.1016/j.diabres.2009.10.007, indexed in Pubmed: 19896746.

2. Fox CS, Evans JC, Larson MG, et al. Temporal trends in coronary heart disease mortality and sudden cardiac death from 1950 to 1999: the Framingham Heart Study. Circulation. 2004; 110(5): 522-527, doi: 10.1161/01.CIR.0000136993.34344.41, indexed in Pubmed: 15262842.

3. Haffner SM, Lehto S, Rönnemaa T, et al. Mortality from coronary heart disease in subjects with type 2 diabetes and in nondiabetic subjects with and without prior myocardial infarction. N Engl J Med. 1998; 339(4): 229-234, doi: 10.1056/ NEJM199807233390404, indexed in Pubmed: 9673301.

4. MacDonald MR, Petrie MC, Varyani F, et al. Impact of diabetes on outcomes in patients with low and preserved ejection fraction heart failure: an analysis of the Candesartan in Heart failure: Assessment of Reduction in Mortality and morbidity (CHARM) programme. Eur Heart J. 2008; 29(11): 1377-1385, doi: 10.1093/ eurheartj/ehn153, indexed in Pubmed: 18413309.

5. Junttila MJ, Barthel P, Myerburg RJ, et al. Sudden cardiac death after myocardial infarction in patients with type 2 diabetes. Heart Rhythm. 2010; 7(10): 1396-1403, doi: 10.1016/j. hrthm.2010.07.031, indexed in Pubmed: 20682359.

6. Curb JD, Rodriguez BL, Burchfiel CM, et al. Sudden death, impaired glucose tolerance, and diabetes in Japanese American men. Circulation. 1995; 91(10): 2591-2595, doi: 10.1161/01. cir.91.10.2591, indexed in Pubmed: 7743621.

7. Escobedo LG, Caspersen CJ. Risk factors for sudden coronary death in the United States. Epidemiology. 1997; 8(2): 175-180, doi: 10.1097/00001648-199703000-00009, indexed in Pubmed: 9229210.

8. Sexton PT, Walsh J, Jamrozik K, et al. Risk factors for sudden unexpected cardiac death in Tasmanian men. Aust N Z J Med. 1997; 27(1): 45-50, doi: 10.1111/j.1445-5994.1997.tb00913.x, indexed in Pubmed: 9079253.

9. Kannel WB, Wilson PW, D'Agostino RB, et al. Sudden coronary death in women. Am Heart J. 1998; 136(2): 205-212, doi: 10.1053/hj.1998.v136.90226, indexed in Pubmed: 9704680.

10. Balkau B, Jouven X, Ducimetière P, et al. Diabetes as a risk factor for sudden death. Lancet. 1999; 354(9194): 1968-1969, doi: 10.1016/s0140-6736(99)04383-4.

11. Jouven X, Desnos M, Guerot C, et al. Predicting sudden death in the population: the Paris Prospective Study I. Circulation. 1999;
99(15): 1978-1983, doi: 10.1161/01.cir.99.15.1978, indexed in Pubmed: 10209001.

12. Albert CM, Chae CU, Grodstein F, et al. Prospective study of sudden cardiac death among women in the United States. Circulation. 2003; 107(16): 2096-2101, doi: 10.1161/01.CIR.0000065223. 21530.11, indexed in Pubmed: 12695299.

13. Jouven X, Lemaitre RN, Rea TD, et al. Diabetes, glucose level, and risk of sudden cardiac death. Eur Heart J. 2005; 26(20): 2142-2147, doi: 10.1093/eurheartj/ehi376, indexed in Pubmed: 15980034 .

14. Gerstein HC, Miller ME, Byington RP, et al. Effects of intensive glucose lowering in type 2 diabetes. N Engl J Med. 2008; 358(24): 2545-2559, doi: 10.1056/NEJMoa0802743, indexed in Pubmed: 18539917.

15. Cushman WC, Evans GW, Byington RP, et al. ACCORD Study Group. Effects of intensive blood-pressure control in type 2 diabetes mellitus. N Engl J Med. 2010; 362(17): 1575-1585, doi: 10.1056/NEJMoa1001286, indexed in Pubmed: 20228401.

16. Ginsberg HN, Elam MB, Lovato LC, et al. ACCORD Study Group. Effects of combination lipid therapy in type 2 diabetes mellitus. N Engl J Med. 2010; 362(17): 1563-1574, doi: 10.1056/ NEJMoa1001282, indexed in Pubmed: 20228404.

17. Ewing DJ, Campbell IW, Clarke BF. The natural history of diabetic autonomic neuropathy. Q J Med. 1980, doi: 10.1093/oxfordjournals.qjmed.a067610.

18. Rathmann W, Ziegler D, Jahnke M, et al. Mortality in diabetic patients with cardiovascular autonomic neuropathy. Diabet Med. 1993; 10(9): 820-824, doi: 10.1111/j.1464-5491.1993.tb00173.x, indexed in Pubmed: 8281726.

19. Pop-Busui R. Cardiac autonomic neuropathy in diabetes: a clinical perspective. Diabetes Care. 2010; 33(2): 434-441, doi: 10.2337/dc09-1294, indexed in Pubmed: 20103559.

20. Banthia S, Bergner DW, Chicos AB, et al. Detection of cardiovascular autonomic neuropathy using exercise testing in patients with type 2 diabetes mellitus. J Diabetes Complications. 2013; 27(1): 64-69, doi: 10.1016/j.jdiacomp.2012.09.002, indexed in Pubmed: 23083925.

21. Albert CM, Mittleman MA, Chae CU, et al. Triggering of sudden death from cardiac causes by vigorous exertion. N Engl J Med. 2000; 343(19): 1355-1361, doi: 10.1056/NEJM200011093431902, indexed in Pubmed: 11070099.

22. Chicos AB, Kannankeril PJ, Kadish AH, et al. Parasympathetic effects on cardiac electrophysiology during exercise and recovery in patients with left ventricular dysfunction. Am J Physiol Heart Circ Physiol. 2009; 297(2): H743-H749, doi: 10.1152/ ajpheart.00193.2009, indexed in Pubmed: 19525382.

23. Kannankeril PJ, Goldberger JJ. Parasympathetic effects on cardiac electrophysiology during exercise and recovery. Am J Physiol Heart Circ Physiol. 2002; 282(6): H2091-H2098, doi: 10.1152/ ajpheart.00825.2001, indexed in Pubmed: 12003816.

24. Kannankeril PJ, Le FK, Kadish AH, et al. Parasympathetic effects on heart rate recovery after exercise. J Investig Med. 2004; 52(6): 394-401, doi: 10.1136/jim-52-06-34, indexed in Pubmed: 15612453.

25. Goldberger JJ, Le FK, Lahiri M, et al. Assessment of parasympathetic reactivation after exercise. Am J Physiol Heart Circ Physiol. 2006; 290(6): H2446-H2452, doi: 10.1152/ajpheart.01118.2005, indexed in Pubmed: 16415073.

26. Wang NC, Chicos A, Banthia S, et al. Persistent sympathoexcitation long after submaximal exercise in subjects with and without 
coronary artery disease. Am J Physiol Heart Circ Physiol. 2011; 301(3): H912-H920, doi: 10.1152/ajpheart.00148.2011, indexed in Pubmed: 21666114.

27. White DW, Raven PB. Autonomic neural control of heart rate during dynamic exercise: revisited. J Physiol. 2014; 592(12): 2491-2500, doi: 10.1113/jphysiol.2014.271858, indexed in Pubmed: 24756637.

28. Pelchovitz DJ, Ng J, Chicos AB, et al. QT-RR hysteresis is caused by differential autonomic states during exercise and recovery. Am J Physiol Heart Circ Physiol. 2012; 302(12): H2567-H2573, doi: 10.1152/ajpheart.00041.2012, indexed in Pubmed: 22542617.

29. Valensi P, Pariès J, Attali JR. Cardiac autonomic neuropathy in diabetic patients: influence of diabetes duration, obesity, and microangiopathic complications-the french multicenter study. Metabolism. 2003; 52(7): 815-820, doi: 10.1016/s00260495(03)00095-7.

30. Stettler C, Bearth A, Allemann S, et al. QTc interval and resting heart rate as long-term predictors of mortality in type 1 and type 2 diabetes mellitus: a 23-year follow-up. Diabetologia. 2007; 50(1): 186-194, doi: 10.1007/s00125-006-0483-1, indexed in Pubmed: 17096116.

31. American Diabetes Association. Standards of medical care in diabetes - 2018. Diabetes Care. 2018; 41(Suppl 1): S13-27.

32. Lunetta M, Le Moli R, Grasso G, et al. A simplified diagnostic test for ambulatory screening of peripheral diabetic neuropathy. Diabetes Res Clin Pract. 1998; 39(3): 165-172, doi: 10.1016/ s0168-8227(98)00005-9, indexed in Pubmed: 9649948.

33. Ewing DJ, Martyn CN, Young RJ, et al. The value of cardiovascular autonomic function tests: 10 years experience in diabetes. Diabetes Care. 1985; 8(5): 491-498, doi: 10.2337/diacare.8.5.491, indexed in Pubmed: 4053936.

34. Maciel BC, Gallo L, Marin-Neto JA, et al. Efficacy of pharmacological blockade of the cardiac parasympathetic system with atropine in normal men. Braz J Med Biol Res. 1985; 18(3): 303-308, indexed in Pubmed: 3835981.

35. Jose AD. Effect of combined sympathetic and parasympathetic blockade on heart rate and cardiac function in man. Am J Cardiol. 1966; 18(3): 476-478, doi: 10.1016/0002-9149(66)90073-7, indexed in Pubmed: 5922892.

36. Johnson NP, Goldberger JJ. Prognostic value of late heart rate recovery after treadmill exercise. Am J Cardiol. 2012; 110(1): 45-49, doi: 10.1016/j.amjcard.2012.02.046, indexed in Pubmed: 22463837.

37. Ng J, Sundaram S, Kadish AH, et al. Autonomic effects on the spectral analysis of heart rate variability after exercise. Am J Physiol Heart Circ Physiol. 2009; 297(4): H1421-H1428, doi: 10.1152/ajpheart.00217.2009, indexed in Pubmed: 19648255.
38. Heart rate variability: standards of measurement, physiological interpretation and clinical use. Task Force of the European Society of Cardiology and the North American Society of Pacing and Electrophysiology. Circulation. 1996; 93(5): 1043-1065, indexed in Pubmed: 8598068.

39. Batchvarov VN, Ghuran A, Smetana P, et al. QT-RR relationship in healthy subjects exhibits substantial intersubject variability and high intrasubject stability. Am J Physiol Heart Circ Physiol. 2002; 282(6): H2356-H2363, doi: 10.1152/ajpheart.00860.2001, indexed in Pubmed: 12003846.

40. Johnson NP, Holly TA, Goldberger JJ. QT dynamics early after exercise as a predictor of mortality. Heart Rhythm. 2010; 7(8): 1077-1084, doi: 10.1016/j.hrthm.2010.05.018, indexed in Pubmed: 20478405 .

41. Burger AJ, Weinrauch LA, D'Elia JA, et al. Effect of glycemic control on heart rate variability in type I diabetic patients with cardiac autonomic neuropathy. Am J Cardiol. 1999; 84(6): 687691, doi: 10.1016/s0002-9149(99)00417-8, indexed in Pubmed: 10498140.

42. Howorka K, Pumprla J, Haber P, et al. Effects of physical training on heart rate variability in diabetic patients with various degrees of cardiovascular autonomic neuropathy. Cardiovasc Res. 1997; 34(1): 206-214, doi: 10.1016/s0008-6363(97)00040-0, indexed in Pubmed: 9217892.

43. Didangelos TP, Arsos GA, Karamitsos DT, et al. Effect of quinapril or losartan alone and in combination on left ventricular systolic and diastolic functions in asymptomatic patients with diabetic autonomic neuropathy. J Diabetes Complications. 2006; 20(1): 1-7, doi: 10.1016/j.jdiacomp.2005.05.002, indexed in Pubmed: 16389160.

44. Bergner DW, Goldberger JJ. Diabetes mellitus and sudden cardiac death: what are the data? Cardiol J. 2010; 17(2): 117-129, indexed in Pubmed: 20544609.

45. Vaduganathan M, Claggett BL, Chatterjee NA, et al. Sudden death in heart failure with preserved ejection fraction: a competing risks analysis from the TOPCAT trial. JACC Heart Fail. 2018; 6(8): 653-661, doi: 10.1016/j.jchf.2018.02.014, indexed in Pubmed: 29501806.

46. Paolisso G, Manzella D, Tagliamonte MR, et al. Effects of different insulin infusion rates on heart rate variability in lean and obese subjects. Metabolism. 1999; 48(6): 755-762, doi: 10.1016/ s0026-0495(99)90176-2, indexed in Pubmed: 10381151.

47. Muta K, Morgan DA, Rahmouni K. The role of hypothalamic mTORC1 signaling in insulin regulation of food intake, body weight, and sympathetic nerve activity in male mice. Endocrinology. 2015; 156(4): 1398-1407, doi: 10.1210/en.2014-1660, indexed in Pubmed: 25574706. 